\title{
Incidencia, supervivencia y factores de riesgo del recién nacido con extremo bajo peso en un hospital
}

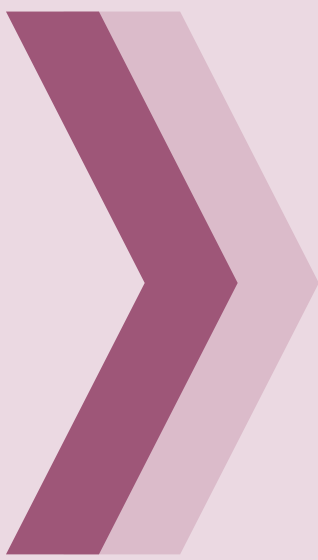

\author{
Impact, survival and \\ risk factors end of newborn \\ extremely low weight in \\ a hospital
}

\author{
Manuel Ticona-Rendon, ${ }^{1, a}$ \\ Diana Huanco-Apaza., \\ Diana Ticona-Huanco $0^{3, c}$
}

1 Médico pediatra y neonatólogo. Doctor en Medicina y Salud Pública. Facultad de Ciencias de la Salud. Universidad Nacional Jorge Basadre de Tacna.

2 Obstetra. Doctora en Salud Pública. Hospital Hipólito Unanue, Tacna.

3 Estudiante de Medicina. Universidad Privada de Tacna.

\section{RESUMEN}

Овлетіvo. Conocer la incidencia, supervivencia y factores de riesgo del recién nacido con extremo bajo peso (RNEBP) en el Hospital Hipólito Unanue, de Tacna (HHUT). PACIENTES Y MÉtodo. Estudio retrospectivo analítico de casos y controles. Se analizaron a todos los nacidos vivos de embarazo único, con peso al nacer menor de $1000 \mathrm{~g}$, nacidos durante los años 2000 a 2014 en el HHUT. Se evaluó la incidencia y supervivencia por 100 nacidos vivos y se analizaron los factores de riesgo. Los casos fueron todos los recién nacidos con peso $<1000 \mathrm{~g}$ y los controles, todos los recién nacidos con peso entre $2500 \mathrm{y}$ $3999 \mathrm{~g}$. Se calculó el odds ratio (OR), con un intervalo de confianza del $95 \%$. La información se obtuvo de la base de datos del Sistema Informático Perinatal. Resultados. De 49979 recién nacidos vivos, 191 tuvieron peso < 1000 g; la incidencia de RNEBP fue 0,38 por 100 nacidos vivos y la supervivencia, 19,7\%, ambas con tendencia ascendente. Los factores de riesgo asociados fueron: madre soltera $(O R=1,9)$, ausencia o control prenatal inadecuado $(O R=11,6)$ y presencia de enfermedades maternas $(O R=2,5)$, tales como como amenaza de parto prematuro $(O R=24,9)$, rotura prematura de membranas $(O R=8,1)$, alteraciones del líquido amniótico $(O R=7,5)$, hemorragia del tercer trimestre $(O R=5,3)$ y enfermedad hipertensiva del embarazo $(O R=4,2)$. Conclusiones. El RNEBP en el HHUT, tiene baja incidencia y supervivencia; sus factores de riesgo son: la falta o inadecuado control prenatal y las enfermedades maternas.

Palabras clave: peso al nacer; recién nacido; bajo peso; muy bajo peso; peso extremadamente bajo; mortalidad neonatal precoz

\begin{abstract}
Oвjective. To determine the incidence, risk factors and survival of newborn with extremely low weight in the Hipolito Unanue Hospital, in Tacna. Patients and METHOD. A retrospective, analytical, case-control study. All live births of singleton pregnancy with birth weight less than 1,000 g, born during the years 2000-2014 in the Hipolito Unanue Hospital in Tacna, Peru, were analyzed. The incidence and survival per 100 live births was evaluated and risk factors were analyzed. The cases were all infants less than $1000 \mathrm{~g}$, and controls, all newborns weighing between 2500 and $3999 \mathrm{~g}$. The odds ratio (OR)was calculated with a confidence interval of $95 \%$. The information was obtained from the database of Perinatal Information System. Results. 49979, 191 live births were born with less than 1000 grams of weight, the incidence of infants of extremely low birth weight was 0,38 x 100 live births and survival 19,7\%, with upward trends. The associated risk factors were significantly: single mother $(O R=1,9)$, no or inadequate prenatal care $(O R=11,6)$, presence of maternal pathologies $(O R=2,5)$ and preterm delivery $(O R=24,9)$, premature rupture of membranes $(O R=8,1)$, abnormal amniotic fluid $(O R=7,5)$, third trimester bleeding $(O R=$ $5,3)$ and hypertensive disease of pregnancy $(O R=4,2)$. Conclusion. The newly born extremely low birth weight Hipolito Unanue Hospital in Tacna has low incidence and survival, and its risk factors lack or inadequate prenatal and maternal pathologies.
\end{abstract}

KEY WORDs: birth weight; infant; birth weight; low weight; very low weight; extremely 


\section{INTRODUCCIÓN}

En la literatura se define al recién nacido con extremo bajo peso (RN-EBP) como aquellos con menos de $1000 \mathrm{~g}$ al nacer. La contribución de este hecho a la morbilidad y la mortalidad son altas, debido a las características fisiológicas y anatómicas de estos, entre ellas: la deficiente termorregulación, la dificultad en su nutrición, necesidades especiales de líquidos y electrolitos, el deficiente control metabólico, la mayor susceptibilidad a las infecciones, entre otros. ${ }^{1}$

Uno de los desafíos más importantes de la neonatología en los últimos años, y en años por venir, en el manejo integral del recién nacido (RN) muy pequeño, es lograr disminuir la elevada tasa de mortalidad en los niños que nacen con extremado bajo peso y también su morbilidad a largo plazo, la cual es una área aún no resuelta de la medicina perinatal. Si bien esta tasa representa un bajísimo porcentaje de todos los nacimientos (menor de $0,7 \%$ ), su contribución a la mortalidad infantil es altísima: entre $20 \%$ y $50 \%$ de todos los niños que fallecen antes de un año de vida. ${ }^{2}$

Sin duda, lo ideal en un futuro sería prevenir el nacimiento de niños con extremo bajo peso, aunque sea prolongando la edad gestacional por dos o tres semanas, para que los niños de pretérmino nazcan después de las 28 semanas cumplidas de duración del embarazo. Esto todavía no se ha podido lograr, incluso con el aumento de cuidados prenatales, el uso de tocolíticos ni con las unidades de cuidados especiales materno-fetales. Hasta que esto se logre, los especialistas en medicina materno-fetalneonatal tendrán que seguir enfrentando el desafío de tratar no solo a los niños de extremado bajo peso, sino también a sus madres y familiares. ${ }^{2}$

El abordaje de estos neonatos ha sido objeto de numerosos estudios, en los que se involucraron no solo los neonatólogos, sino también obstetras y comités de bioética. El primer paso para llegar al mismo es conocer la situación de estos pacientes en el mundo, en el país y, finalmente, en nuestra región. ${ }^{3}$ Sin embargo, la sobrevida de estos RN ha aumentado considerablemente en las últimas décadas con el desarrollo de los cuidados intensivos neonatales y los avances tecnológicos, principalmente con relación a la ventilación asistida. Quizás el mayor impacto en el manejo neonatal haya sido la administración prenatal de corticoides y el surfactante pulmonar exógeno en el manejo del síndrome de dificultad respiratoria. ${ }^{4}$

Existen diferencias en las cifras de sobrevida de estos pacientes entre un país y otro, debido a las diferencias en la formación de recursos humanos, las prácticas clínicas y la tecnología. Dentro de un país, la mortalidad en determinado subgrupo varía de un hospital a otro. Hay evidencias que demuestran que los RN con EBP tienen mayor sobrevida si son atendidos en unidades de mayor nivel y con mayor volumen de pacientes. ${ }^{5}$ La natalidad del $\mathrm{RN}$ con EBP ha sido variable e infrecuente; sin embargo, su supervivencia como indicador ha mejorado, pero se requiere de un manejo especializado para evitar las múltiples secuelas que pueden sobrevenir, especialmente las neurológicas y respiratorias. Es de interés conocer qué ha sucedido en los últimos quince años en el Hospital Hipólito Unanue, de Tacna (HHUT), en cuanto a términos de incidencia y supervivencia, con la finalidad de mejorarlos y lograr no solo la supervivencia, sino también, una mejor calidad de vida, aspecto pendiente de resolver. El objetivo de este estudio fue conocer los factores de riesgo asociados del RN con extremo bajo peso, con la finalidad de disminuir la incidencia y la mortalidad de este grupo.

\section{MATERIALES Y MÉTODOS}

Estudio retrospectivo, analítico, de casos y controles. La población de estudio estuvo conformada por la totalidad de RN vivos de embarazo único, con peso al nacer menor de $1000 \mathrm{~g}$, nacidos durante los años 2000 a 2014, en el HHUT. Para el análisis de factores de riesgo se conformaron dos grupos: los casos o grupo de estudio (los $191 \mathrm{RN}$ con peso al nacer menor de $1000 \mathrm{~g}$ ) y los controles (41 $296 \mathrm{RN}$ con peso entre 2500 y $3999 \mathrm{~g})$.

La información se obtuvo de la base de datos del Sistema Informático Perinatal. Para el análisis de la incidencia y supervivencia se utilizó la frecuencia por cien nacidos vivos. Para identificar los factores de riesgo se realizó el análisis bivariado, comparando el grupo estudio con el grupo control; se calculó el odds ratio, con un intervalo de confianza de $95 \%$; se consideró significativo cuando el valor fue mayor de 1 y p menor de 0,05 . En el procesamiento y análisis de datos se utilizaron los programas SIP2000 y Epi Info. 


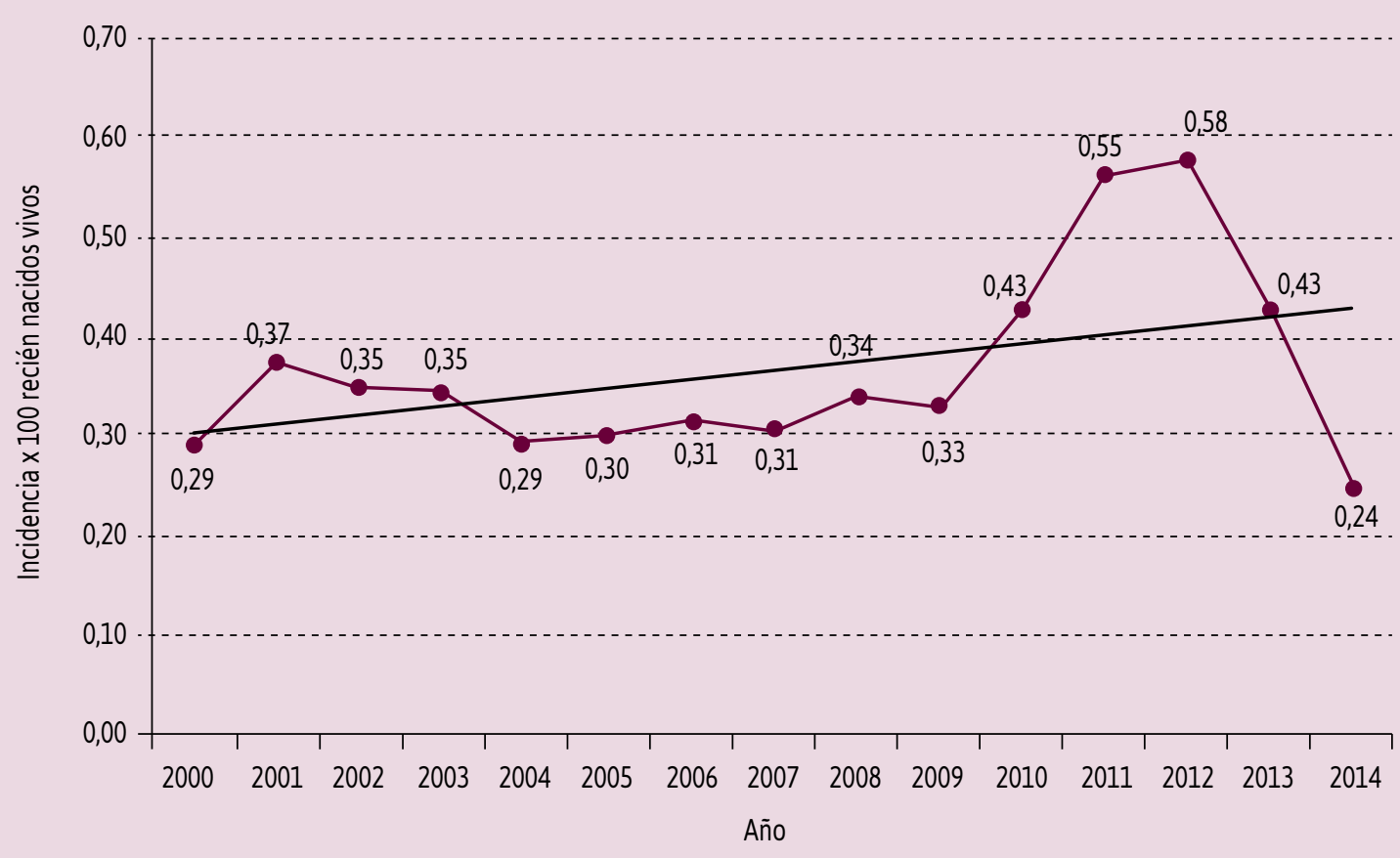

Figura 1. Tendencia de la incidencia de los recién nacidos con extremo bajo peso. Hospital Hipólito Unanue, de Tacna, 2000-2014

\section{RESULTADOS}

Durante los años 2000 a 2014 se atendieron 49979 partos con RN vivos, de los cuales 191 pesaron de 500 a $999 \mathrm{~g}$ al nacer; es decir, la incidencia global promedio de RN-EBP fue $0,38 \%$ por 100 nacidos vivos, con valores entre $0,24 \%$ y $0,58 \%$, con una tendencia ascendente durante los quince años de estudio, sin existir diferencias significativas $(p=$ 0,65 ) (Figura 1).

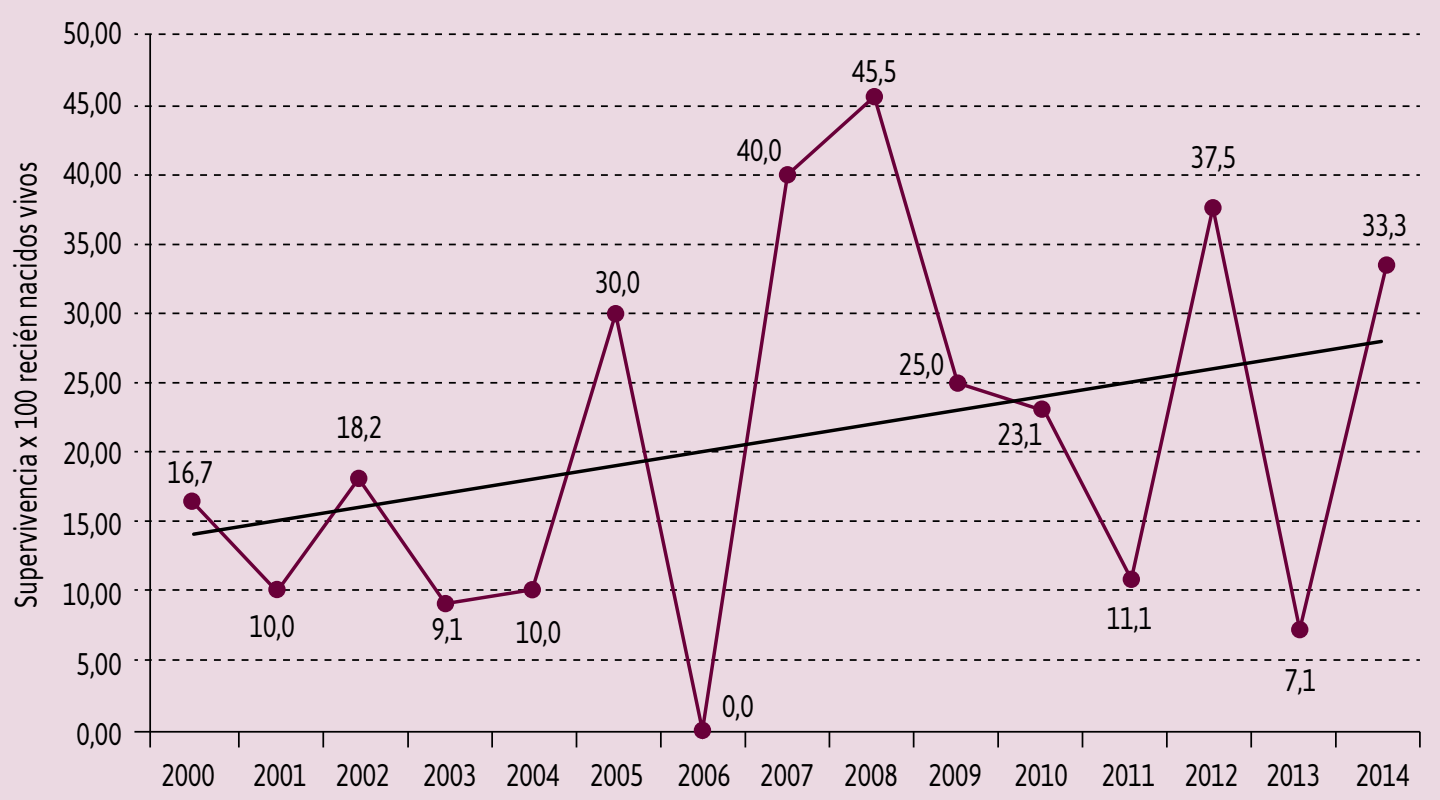

Figura 2. Supervivencia de los recién nacidos con extremo bajo peso. Hospital Hipólito Unanue, de Tacna, 2000-2014 
Tabla 1. Factores de riesgo socioeconómicos para los recién nacidos de extremo bajo peso en el Hospital Hipólito Unanue, de Tacna, 2000-2014.

\begin{tabular}{|c|c|c|c|c|c|c|}
\hline \multirow[t]{2}{*}{ Factores socioeconómicos } & \multicolumn{2}{|c|}{ Casos } & \multicolumn{2}{|c|}{ Controles } & \multirow[t]{2}{*}{ OR } & \multirow[t]{2}{*}{ IC } \\
\hline & $\mathrm{N}$ & $\%$ & $\mathrm{~N}$ & $\%$ & & \\
\hline \multicolumn{7}{|l|}{$\Delta$ Estado civil } \\
\hline - Casada & 23 & 12,04 & 6598 & 16,03 & Referencia & \\
\hline - Conviviente & 126 & 65,97 & 28204 & 68,51 & 1,28 & $0,81-2,05$ \\
\hline - Soltera & 41 & 21,47 & 6302 & 15,31 & 1,87 & $1,09-3,21$ \\
\hline - Viuda o divorciada & 1 & 0,52 & 63 & 0,15 & 4,55 & $0,62-32,81$ \\
\hline \multicolumn{7}{|l|}{$\Delta$ Grado instrucción } \\
\hline - Analfabeta & 1 & 0,53 & 158 & 0,38 & 1,35 & $0,19-9,59$ \\
\hline - Primaria & 24 & 12,63 & 4836 & 11,75 & 1,06 & $0,67-1,67$ \\
\hline - Secundaria & 128 & 67,37 & 27312 & 66,35 & Referencia & \\
\hline - Superior & 37 & 19,47 & 8855 & 21,51 & 0,89 & $0,61-1,30$ \\
\hline
\end{tabular}

La tasa de supervivencia promedio de estos RN durante los quince años de estudio fue de $19,7 \%$, con valores entre 0 y $45,5 \%$, pero sin diferencias significativas $(p=0,52)$ (Figura 2).

Del total de RN fallecidos durante los años 2000 a 2014, 24,4\% fueron RN-EBP; es decir, que de cada cuatro muertes neonatales, uno tenía menos de $1000 \mathrm{~g}$. El único factor de riesgo socioeconómico asociado significativamente fue "madre soltera» $(\mathrm{OR}=1,9)($ Tabla 1$)$. No se presentó ningún factor de riesgo biológico ni obstétrico asociado con el extremo bajo peso al nacer (Tablas 2 y 3 ). Los factores de riesgo durante la evolución del embarazo y parto asociados con el RN-EBP fueron ausencia o control prenatal inadecuado $(O R=11,6)$ y presencia de enfermedades maternas $(O R=2,5)$, como amenaza de parto prematuro $(O R=24,9)$, rotura prematura de membranas $(O R=8,1)$, alteraciones del líquido amniótico $(O R=7,5)$, hemorragia del tercer trimestre $(O R=5,3)$ y enfermedad hipertensiva del embarazo $(O R=4,2)$ (Tablas 4 y 5 ).

Tabla 2. Factores de riesgo biológicos para recién nacido de extremo bajo peso en el Hospital Hipólito Unanue de Tacna. 2000-2014

\begin{tabular}{|c|c|c|c|c|c|c|}
\hline \multirow[t]{2}{*}{ Factores biológicos } & \multicolumn{2}{|c|}{ Casos } & \multicolumn{2}{|c|}{ Controles } & \multirow[t]{2}{*}{ OR } & \multirow[t]{2}{*}{ IC } \\
\hline & $\mathrm{N}$ & $\%$ & $\mathrm{~N}$ & $\%$ & & \\
\hline$\Delta$ Edad materna & 191 & & 41277 & & & \\
\hline - Menor de 20 & 31 & 16,23 & 7345 & 17,79 & 0,94 & $0,63-1,42$ \\
\hline - De 20 a 34 & 132 & 69,11 & 29519 & 71,51 & Referencia & \\
\hline - De 35 a más & 28 & 14,66 & 4413 & 10,69 & 1,42 & $0,92-2,17$ \\
\hline$\Delta$ Índice masa corporal & 191 & & & & & \\
\hline - Menos de 18,5 & 3 & 1,57 & 640 & 1,55 & 0,95 & $0,24-3,11$ \\
\hline - De 18,5 a 24,9 & 109 & 57,07 & 22156 & 53,65 & Referencia & \\
\hline - De 25 a 29,9 & 60 & 31,41 & 13774 & 33,35 & 0,89 & $0,64-1,23$ \\
\hline - De 30 a más & 19 & 9,95 & 4726 & 11,44 & 0,82 & $0,49-1,36$ \\
\hline \multicolumn{7}{|c|}{$\triangle$ Antecedente materno de BPN } \\
\hline - Sí & 1 & 0,52 & 34 & 0,08 & 6,39 & $0,90-43,25$ \\
\hline- No & 190 & 99,48 & 41262 & 99,92 & Referencia & \\
\hline
\end{tabular}


Tabla 3. Factores de riesgo obstétricos asociados a recién nacido de extremo bajo peso en el Hospital Hipólito Unanue de Tacna. 2000-2014

\begin{tabular}{|c|c|c|c|c|c|}
\hline \multirow[t]{2}{*}{ Factores socioeconómicos } & \multicolumn{2}{|c|}{ Casos } & \multicolumn{2}{|c|}{ Controles } & \multirow[t]{2}{*}{ OR } \\
\hline & $\mathrm{N}$ & $\%$ & $\mathrm{~N}$ & $\%$ & \\
\hline$\triangle$ Paridad & & & 41296 & & \\
\hline - Primípara & 85 & 44,50 & 19859 & 48,09 & $0,910,67-1,25$ \\
\hline - Multípara & 84 & 43,98 & 17910 & 43,37 & Referencia \\
\hline - Gran multípara & 22 & 11,52 & 3527 & 8,54 & $1,330,81-2,17$ \\
\hline \multicolumn{6}{|l|}{$\Delta$ Periodo intergenésico } \\
\hline - Menor de 12 meses & 30 & 28,30 & 4062 & 18,95 & $1,440,84-2,47$ \\
\hline - 12 a 24 meses & 14 & 13,21 & 3385 & 15,79 & $0,800,40-1,58$ \\
\hline - 25 a 48 meses & 29 & 27,36 & 5641 & 26,31 & Referencia \\
\hline - Mayor de 48 meses & 33 & 31,13 & 8349 & 38,95 & $0,770,45-1,30$ \\
\hline \multicolumn{6}{|l|}{$\triangle$ Antecedente hijo de BPN } \\
\hline - Sí & 4 & 2,09 & 326 & 0,79 & $2,690,85-7,54$ \\
\hline - No & 187 & 97,91 & 40970 & 99,21 & Referencia \\
\hline
\end{tabular}

Tabla 4. Factores de riesgo de la gestación y parto para recién nacido de extremo bajo peso en el Hospital Hipólito Unanue de Tacna. 2000-2014

\begin{tabular}{|c|c|c|c|c|c|c|}
\hline \multirow[t]{2}{*}{ Factores biológicos } & \multicolumn{2}{|c|}{ Casos } & \multicolumn{2}{|c|}{ Controles } & \multirow[t]{2}{*}{ OR } & \multirow[t]{2}{*}{ IC } \\
\hline & $\mathrm{N}$ & $\%$ & $\mathrm{~N}$ & $\%$ & & \\
\hline$\Delta$ Control prenatal & & & 41296 & & & \\
\hline-0 & 88 & 46,07 & 6026 & 14,59 & 12,20 & $8,14-18,34$ \\
\hline$-1-5$ & 67 & 35,08 & 5187 & 12,56 & 10,79 & $7,07-16,53$ \\
\hline - 6 a más & 36 & 18,85 & 30083 & 72,85 & Referencia & \\
\hline$\triangle$ Primer CPN & & & 35270 & & & \\
\hline - I trimestre (1-13 sem) & 44 & 42,72 & 13874 & 39,34 & Referencia & \\
\hline - II trimestre (14-27 sem,) & 56 & 54,37 & 13136 & 37,24 & 1,34 & $0,89-2,03$ \\
\hline - III trimestre (28 a más) & 3 & 2,91 & 8260 & 23,42 & 0,11 & $0,03-0,38$ \\
\hline \multicolumn{7}{|l|}{$\Delta$ Enfermedad materna } \\
\hline - Sí & 139 & 72,77 & 21478 & 52,01 & 2,47 & $1,77-3,44$ \\
\hline- No & 52 & 27,23 & 19818 & 47,99 & Referencia & \\
\hline \multicolumn{7}{|l|}{$\Delta$ Enfermedad materna } \\
\hline - Amenaza de parto prematuro & 78 & 40,84 & 1117 & 2,70 & 24,83 & $18,30-33,67$ \\
\hline - Anemia & 48 & 25,13 & 13174 & 31,90 & 0,72 & 0,51-1,01 \\
\hline - Infección urinaria & 40 & 20,94 & 8680 & 21,02 & 1,00 & $0,69-1,43$ \\
\hline - Enfermedad hipertensiva embarazo & 13 & 6,81 & 700 & 1,70 & 4,24 & $2,29-7,66$ \\
\hline - Rotura prematura de membranas & 10 & 5,24 & 281 & 0,68 & 8,06 & $3,98-15,86$ \\
\hline - Hemorragia III trimestre & 5 & 2,62 & 210 & 0,51 & 5,26 & $1,89-13,40$ \\
\hline - Amenaza de aborto & 3 & 1,57 & 468 & 1,13 & 1,39 & $0,35-4,51$ \\
\hline - Alteraciones líquidp amniótico & 3 & 1,57 & 88 & 0,21 & 7,47 & $1,87-24,68$ \\
\hline - Enfermedades crónicas & 0 & 0,00 & 155 & 0,38 & & \\
\hline - Terminación & 191 & & 41243 & & & \\
\hline - Cesárea & 60 & 31,41 & 13095 & 31,75 & 0,98 & $0,72-1,35$ \\
\hline - Vaginal & 131 & 68,59 & 28148 & 68,25 & Referencia & \\
\hline
\end{tabular}


Tabla 5. Factores de riesgo asociados a recién nacido de extremo bajo peso en el Hospital Hipólito Unanue de Tacna. 2000-2014

Factores

OR

IC

$\triangle$ Amenaza de parto prematuro (APP)

- Con APP

$24,8718,30-33,67$

- Sin APP

$\triangle$ Control prenatal

$-0-5$

$11,55 \quad 7,93-16,90$

- 6 a más

$\triangle$ Rotura prematura de membranas (RPM)

- Con RPM $8,06 \quad 3,98-15,86$

- Sin RPM

$\triangle$ Alteraciones del líquido amniótico

- Con alteraciones

$7,47 \quad 1,87-24,68$

- Sin alteraciones

$\Delta$ Hemorragia del tercer trimestre

- Con hemorragia

$5,26 \quad 1,89-13,40$

- Sin hemorragia

$\triangle$ Enfermedad hipertensiva embarazo (EHE)

- Con EHE

$4,24 \quad 2,29-7,66$

- Sin EHE

$\Delta$ Enfermedad materna

- Con enfermedad

$2,47 \quad 1,77-3,44$

- Sin enfermedad

$\triangle$ Estado civil

- Soltera

$1,87 \quad 1,09-3,21$

- Casada/conviviente en el Instituto Hondureño de la Seguridad Social, de 1999 al 2001 (0,3\%), y Salas et al., ${ }^{8}$ en el Hospital Barros Luco, de Santiago, en los años 2000 a 2004 (0,34\%). Incidencias mayores encontraron: Hinojosa et al., ${ }^{9}$ en el Hospital Infantil de México, en el periodo comprendido entre mayo 1985 y abril de 1986, donde de 23457 RN vivos 0,5\% tuvieron menos de 1000 g; Padilla et al., ${ }^{10}$ en el Hospital de Gineco Obstetricia N.$^{\circ} 23$ en México, en 20052006, donde de 18952 nacimientos encontró 0,8\% RN-EBP; Cancela et al.,11 en el Centro Hospitalario Pereira Rossell, de Uruguay, en el año 2002, encontró 1\%; Cruz et al.,12 en el Hospital Santo Cecilio de Granada, en España, del 1991 a 2010, de 56024 nacidos durante este periodo, encontró $264 \mathrm{RN}$ con menos de $1000 \mathrm{~g}$, con una incidencia de $0,47 \%$ y Bancalari, ${ }^{13}$ en el Hospital Jackson Memorial, de la Universidad de Miami, de 1977 hasta 2001 , encontró $1,5 \%$ de RN-EBP.

Incidencias menores encontraron: García et al., ${ }^{14}$ en el hospital San Antonio de los Baños (La Habana), en Cuba, durante los años 1986 a 2005, de un total de 40078 nacidos vivos, encontró $0,14 \%$ de $R N$ con peso menor de $1000 \mathrm{~g}$ y una edad gestacional inferior de 28 semanas y Molina et al., ${ }^{1}$ en el Hospital Gineco Obstétrico Docente Mariana Grajales, de Santa Clara, en Cuba, en el periodo del 2000 al 2008, quienes encontraron una incidencia baja de $\operatorname{EBP}(0,07 \%$ del total de nacidos vivos).

Respecto a la sobrevida, solo Saldaña et al. ${ }^{7}$ en Honduras encontró una sobrevida de 10\%, menor que nuestros resultados. La mayoría de los autores encontraron sobrevida mayores a los nuestros, como: Hinojosa et al. ${ }^{9}(24,4 \%)$ y Padilla et al. ${ }^{10}$ $(35,6 \%)$, en México; Cancela et al.,11 en Uruguay (32,3\% en 2002 y $56 \%$ en 2004 ), después de poner en práctica un protocolo para el manejo de estos RN. Salas et al., ${ }^{8}$ en Santiago de Chile (44,8\%); García et al. ${ }^{14}(76,4 \%)$ y Molina et al. ${ }^{1}(70,8 \%)$, en Cuba; Cruz et al.,12 en España (62,6\%). Bancalari, ${ }^{13}$ en Miami, EE. UU., de 1977 a 2001, encontró que la sobrevida se incrementó de $20 \%$ a $40 \%$ hasta $80 \%$ a $90 \%$; esta sobrevida no solo se debe a los avances de la neonatología, sino también a los cambios en manejo obstétrico de las madres de estos niños.

Sola, ${ }^{2}$ en 11 centros de América Latina, encontró que la tasa de sobrevida en menores de $1000 \mathrm{~g}$, varía entre una tasa tan alta de $80 \%$ hasta tan

A nivel internacional, incidencia semejantes también fueron encontradas por: Saldaña et al., 
bajas como de solo $10 \%$ o $20 \%$ de sobrevida. El RN-EBP es un grupo demográfico con bajo índice de sobrevivencia, debido a factores obstétricos y perinatales desfavorables. Los estudios internacionales en esta población muestran una tendencia creciente a mejorar la sobrevida gracias a los avances en cuidados intensivos neonatales. Mesquita et al. ${ }^{3}$ afirman que el aumento de sobrevida de estos pacientes y los límites de viabilidad han ido bajando, de 30 a 31 semanas en la década de 1960, a 24 semanas en la década de 1990.

Cruz et al. ${ }^{12}$ refieren que la sobrevida ha aumentado debido a la mejora en la salud de la población y a la asistencia sanitaria recibida por las gestantes y neonatos y especialmente al desarrollo de nuevas tecnologías para el control del bienestar fetal y de los cuidados neonatales. El nacimiento de un neonato en el límite de viabilidad constituye un dilema ético para el equipo médico, porque deben tomarse decisiones muy importantes, como reanimarlo en sala de partos, brindarle los cuidados intensivos plenos y dar información a los padres. Evidentemente, por los datos citados, los límites de viabilidad de un prematuro extremo en las regiones con mayor desarrollo socioeconómico no son extrapolables a países como los nuestros de menor desarrollo. La sobrevida de los RNEBP aumentó en las últimas décadas con el desarrollo de los cuidados intensivos neonatales y los avances tecnológicos, principalmente los relacionados con la ventilación asistida. Quizás el mayor impacto en el manejo de los mismos haya sido la administración prenatal de corticoides y el surfactante exógeno en el manejo del síndrome de dificultad respiratoria en el prematuro.

Con relación a la ética del profesional de la atención de este grupo tan vulnerable, las nuevas guías de resucitación de la Academia Americana de Pediatría y el International Liaison Committee on Resuscitation (ILCR) incluyen entre sus recomendaciones la participación de los padres en la toma de decisiones para aquellos neonatos que se encuentran en los límites de viabilidad (23 a 25 semanas). ${ }^{15}$ En definitiva, Sola ${ }^{2}$ menciona que muchos de los RN-EBP hoy sobreviven, pero pueden sobrevivir todavía más. Lo importante para lograr este objetivo es brindar atención general, integral y completa, cuidando al máximo todos los detalles, todo el tiempo. Con compasión. Con enfermeras en número suficiente, altamente capacitadas. Junto con ello, es un desafío disminuir las morbilidades y secuelas.

En nuestro estudio la mortalidad de los RN-EBP contribuye en $24,4 \%$ a la mortalidad neonatal en el hospital Hipólito Unanue, de Tacna. Encontraron mayores porcentajes de contribución a la mortalidad neonatal: Saldaña et al., ${ }^{7}$ en Honduras (60\%); Cancela et al.,1 ${ }^{11}$ en Uruguay (40\%); Bancalari, ${ }^{13}$ en Miami (54,8\%); Cruz et al.,12 en Granada, España (31\%), y García et al.,14 en La Habana, Cuba (entre 20 y $50 \%$ ). La contribución a la morbilidad y mortalidad es alta, debido a las características fisiológicas y anatómicas de estos RN, entre ellas: la deficiente termorregulación, la dificultad en su nutrición, las necesidades especiales de líquidos y electrolíticos, el deficiente control metabólico, la falta de madurez orgánica (especialmente pulmonar) y la mayor susceptibilidad a las infecciones.

Luego se analizan los factores de riesgo asociados con los RN-EBP, en comparación con los RN con peso adecuado. Se debe mencionar que no se encontró otro estudio de casos y controles sobre factores de riesgo; únicamente estudios descriptivos, los cuales se mencionan a continuación.

Analizando los factores de riesgo socioeconómicos, el único factor asociado significativamente fue el estado civil «soltera». No se encontró relación con el bajo grado de instrucción.

Lohmann et al., ${ }^{6}$ en el Hospital Nacional Cayetano Heredia, encontraron, en las madres de estos $\mathrm{RN}$, que la mayoría tenía grado de instrucción secundaria y estado civil conviviente, hallazgos semejantes a nuestro estudio. En cuanto a los factores biológicos, no se evidenció asociación de extremo bajo peso al nacer con la edad materna en los extremos de la vida, mal estado nutricional pregestacional (medido con el índice de masa corporal) ni con el antecedente materno de bajo peso. El mismo autor, también en el Hospital Cayetano Heredia, encontró que las características de las madres de estos RN fueron edad materna mayor de 19 y menor de 35 años. Padilla et al., ${ }^{10}$ en México, encontró que los rangos de la edad materna fueron 15 y 34 años, con una media de 25,4 años. Salas et al., ${ }^{8}$ en Chile, encontró que el promedio de edad de las madres fue 26,7 años; 
$25,3 \%$ fueron adolescentes y $16,3 \%$, mayores de 35 años. En nuestro estudio, se encontró un promedio de edad de 26 años; $16,2 \%$ fueron adolescentes y $14,7 \%$, mayores de 34 años.

Conrespectoalosfactoresobstétricos, encontramos que ni la gran multiparidad, periodo intergenésico menor de 12 meses ni el antecedente de hijo con BPN fueron factores de riesgo para el extremo bajo peso al nacer. Lohmann et al., ${ }^{6}$ en el Hospital Cayetano Heredia, encontró que las madres de RN-EBP fueron en su mayoría primigestas, semejante a nuestro estudio. Con relación a los factores de la gestación y parto, se encontró que sí fueron factores de riesgo asociados de manera significativa con el extremo bajo peso al nacer: la ausencia de control prenatal, el control prenatal inadecuado, la presencia de alguna enfermedad materna, la amenaza de parto prematuro, rotura prematura de membranas, alteraciones del líquido amniótico, hemorragia del tercer trimestre y enfermedad hipertensiva del embarazo. No existió asociación con el inicio tardío del control prenatal, anemia, infección urinaria, amenaza de aborto ni la terminación por operación cesárea.

En cuanto al control prenatal, Lohmann encontró diferentes resultados en el Hospital Cayetano Heredia; menciona un control prenatal inadecuado en el $76 \%$. En nuestro estudio, $46,1 \%$ no tuvieron control prenatal y $35,1 \%$ tuvieron control prenatal inadecuado. Al analizar las enfermedades maternas, si bien son estudios descriptivos, la mayoría coincide con nuestros resultados. Así, Lohmann, en el Hospital Cayetano Heredia, encontró que las complicaciones obstétricas más frecuentes fueron rotura prematura de membranas, $28 \%$; hemorragia del tercer trimestre, $15 \%$; preeclampsia grave, $11 \%$ y corioamnioitis, $9 \%$.

Cruz et al. ${ }^{12}$ en el Hospital de Granada, en España, encontró que las causas fundamentales del extremo bajo peso fueron: rotura prematura de membranas, hipertensión materna, amenaza de parto pretérmino incontrolable y gemelaridad. Existen enfermedades maternas o complicaciones propias del embarazo (hipertensión, problemas placentarios, etc.), que son difíciles de disminuir, aunque sí se pueden controlar más exhaustivamente. De hecho, la hipertensión materna es la enfermedad que más contribuye a la mortalidad de estos RN, no solo por la necesidad de tener que provocar un parto pretérmino, sino porque la hipertensión puede producir disfunción placentaria, que ocasiona complicaciones fetales y placentarias, que dan lugar a la muerte fetal 0 neonatal. Hasta ahora, todas las intervenciones para prevenir la hipertensión del embarazo han sido poco eficaces.

Genes et al. ${ }^{16}$ en el Hospital de Clínicas de Asunción, Paraguay, encuentra dentro de las enfermedades más frecuentes la rotura prematura de membranas (37,5\%); hipertensión materna $(25,8 \%)$; retardo de crecimiento intrauterino $(26,6 \%)$ y embarazo múltiple (22,7\%). Refiere que el binomio madre-feto requiere del manejo de un equipo multidisciplinario. Existe suficiente evidencia científica de que el tratamiento antibiótico, cuando se presenta RPM como bacteriuria asintomática e infección de vías urinarias durante el embarazo, disminuyen los partos pretérmino y, por lo tanto, su influencia sobre el peso al nacimiento; al igual que el manejo de la hipertensión arterial, relacionándose en forma directa con la restricción del crecimiento intrauterino. El equipo de obstetricia debería incrementar el porcentaje de tratamiento de estas enfermedades para evitar el nacimiento con extremo bajo peso, que determina una mayor mortalidad neonatal. Padilla et al.,10 en México, encontraron que $7,1 \%$ cursaron con preeclampsi, $3,1 \%$ con corioamnioitis y en $11 \%$ hubo rotura prematura de membranas.

En este estudio encontramos que 31\% terminaron en operación cesárea, porcentaje menor a lo reportado por Genes et al.,16 en Paraguay, y Salas, en el Hospital Sótero del Río, en Santiago de Chile. ${ }^{8}$ Dentro de los cuidados perinatales, el nacimiento del niño con extremo bajo peso mediante la operación cesárea mejora su pronóstico, sobre todo si se acompaña de trabajo de parto previo, pues tienen menos complicaciones y aumenta su sobrevida.

Bancalari, ${ }^{13}$ en Miami, EE. UU., refiere que el efecto de la operación cesárea mejora el pronóstico de estos niños debido a que la incidencia de la hemorragia intracraneal es mayor en niños que nacieron por parto vaginal, en comparación a los nacidos mediante operación cesárea. El efecto protector de la operación cesárea puede deberse a la ausencia de trabajo de parto, o por lo menos 
a un trabajo de parto más breve. El pronóstico de estos RN depende fundamentalmente del trabajo en equipo, porque los resultados de estos niños están en gran parte determinados por las intervenciones que se realizan antes, durante y después del nacimiento.

Salas et al., ${ }^{8}$ en Chile, encontró que 59,4\% de los partos se resolvieron con la operación cesárea, dado que la vía vaginal es un predictor de hemorragia intraventricular severa. Álvarez,, ${ }^{17}$ en Costa Rica, refiere que es evidente que la sobrevida de los pacientes es muy limitada y, por lo tanto, deben realizarse todos los esfuerzos para evitar el nacimiento antes de las 27 semanas de gestación, dentro de las que deben resaltarse el control prenatal adecuado, evitar embarazos en los extremos de la vida, identificar tempranamente los embarazos de alto riesgo y asegurar su vigilancia por personal especializado, detección temprana de la amenaza de parto prematuro y su tratamiento enérgico y refinar las practicas obstétricas para el manejo de parto prematuro.

\section{CONCLUSIONES}

En el Hospital Hipólito Unanue, de Tacna, durante los últimos quince años hay una tendencia a disminuir la incidencia de recién nacidos con extremo bajo peso y mejorar su supervivencia, que actualmente representa la cuarta parte de la mortalidad neonatal.

Los factores de riesgo asociados significativamente con el extremo bajo peso al nacer fueron madre soltera, ausencia o control prenatal inadecuado y presencia de enfermedades maternas, tales como amenaza de parto prematuro, rotura prematura de membranas, alteraciones del líquido amniótico, hemorragia del tercer trimestre y enfermedad hipertensiva del embarazo.

\section{RECOMENDACIONES}

Se recomienda insistir en el control adecuado del riesgo preconcepcional y la atención prenatal de calidad para disminuir la incidencia de recién nacidos con extremo bajo peso, así como la capacitación y perfeccionamiento continuo de los médicos pediatras y obstetras y demás profesionales de la salud relacionados con la atención de estos pacientes, con la finalidad de mejorar no solo la sobrevivencia, sino también su calidad de vida, dada su especial vulnerabilidad. Se sugiere regionalizar los cuidados perinatales. La asistencia del parto del recién nacido con extremo bajo peso se debe realizar en un centro que cuente la infraestructura necesaria y recursos humanos calificados (unidad de cuidados intensivos neonatales) así como con un centro de seguimiento. Finalmente, es necesario contar con protocolos de atención, tanto desde el punto de vista obstétrico como del neonatal.

\section{REFERENCIAS BIBLIOGRÁFICAS}

I. Molina O, Regalado A. Recién nacido de peso extremo. Rev Cubana Obstet Ginecol. 2010;36(2):32-4I.

2. Sola $A$. El recién nacido de extremadamente baja edad gestacional. En: Sola A. Cuidados neonatales: descubriendo la vida de un recién nacido enfermo. Vol 2. Buenos Aires,Argentina: Edimed; 201 I. p. 1337-47.

3. Mesquita M, Lacarrubba J, Galván L, Barreto N, Buena J, Adler E; Comité de Neonatología de la Sociedad Paraguaya de Pediatría. Recién nacidos de extremo bajo peso de nacimiento: límites de viabilidad, reanimación en sala de partos y cuidados intensivos neonatales. Pediatr (Asunción). 2010;37(2):127-35.

4. Harper RG, Rehman KU, Sia C, Buckwald S, Spinazzola R, Schlessel J, et al. Neonatal outcome of infants born at 500 to 800 grams from 1990 through 1998 in a tertiary care center. J Perinatol. 2002;22(7):555-62.

5. Phibbs CS, Baker LC, CaugheyAB, Danielsen B, Schmitt SK, Phibbs $\mathrm{RH}$. Level and volume of neonatal intensive care and mortality in very-low-birth-weight infants. N Engl J Med. 2007;356(2I):2I6575.

6. Lohmann Gandini-Billinghurst P, Rodríguez-Espinosa M, WebbLinares V, Rospigliosi- López $M$. Mortalidad en recién nacidos de extremo bajo peso al nacer en la unidad de Neonatología del Hospital Nacional Cayetano Heredia entre enero 2000 y diciembre 2004. Rev Med Hered. 2006; I7(3): I4I-7.

7. Saldaña M, Pineda E. Mortalidad en neonatos con peso menor de I500 gramos en el servicio de recién nacidos en el Hospital de Especialidades del Instituto Hondureño de Seguridad Social. Rev Med Post UNAH. 2002;7(I):91-6.

8. Salas R, Sanhueza L, Maggi L. Factores de riesgo y seguimiento clínico en prematuros menores de 1000 gramos. Rev Chil Pediatr. 2006;77(6):577-88.

9. Hinojosa J, Reyes R, García R. El recién nacido con peso menor de 1000 gramos: revisión de la morbimortalidad y secuelas a largo plazo. Bol Med Hosp Infant Mex. 1998;45(3): I6I-4.

10. Padilla YM, Hernández RJ. Condiciones asociados a la mortalidad neonatal específica en menores de 1000 gramos, en el Hospital de Gineco-Obstetricia $N^{\circ}$ 23, período 2005-2006. Bol Med Hosp Infant Mex. 2010;67(4): 335-43.

I I. Cancela MJ, Repetto M, Moraes M, Latoff M, Kuzmicz S, Olivera A, et al. Estrategias para disminuir la mortalidad y morbilidad en los recién nacidos menores de 1000 gramos en el sector público (Centro Hospitalario Pereira Rossell, Servicio de Recién Nacidos). Rev Chil Pediatr. 2007;78(4):430-36.

12. Cruz M, Redondo R, CañoA, Carretero P,Padilla C, RuizA.Análisis de la mortalidad perinatal en nacidos con peso menor de 1000 
gramos en el Hospital San Cecilio de Granada (España) durante el período 199|-20 I0.Arch Argent Pediatr. 20 I3; I I ( I):45-52.

13. Bancalari E. Factores perinatales en el pronóstico del prematuro extremo. Arch Pediatr Urug. 2003;74(3):158-65.

14. García Y, Fernández RM. El recién nacido pretérmino extremadamente bajo peso al nacer: un reto a la vida. Rev Cubana Pediatr [Internet]. 2006 [citado 10 oct 2015];78(3):[6 p.]. Disponible en: http://scielo.sld.cu/pdf/ped/v78n3/ped04306. pdf

15. Saugstad OD. New guidelines for newborn resuscitation. Acta Paediatr. 2007;96(3):333-7.

16. Genes L, Lacarrubba J, Caballero C, Fonseca R, Mir R, Céspedes $\mathrm{E}$, et al. Morbi-mortalidad en recién nacidos de muy bajo peso al nacer. Unidad de Neonatalogía. Centro Materno Infantil, Hospital de Clínicas. Pediatr. (Asunción) 201;37(1):9-22.
17. Alvarez J. Morbi-mortalidad de los recien nacidos de muy bajo peso en el Hospital San Juan de Dios, San José, Costa Rica. Acta Pediatr Costarric. 200I;I5(2):46-52.

\section{Correspondencia}

Dr. Manuel Ticona Rendón

manuelticonar@yahoo.es

\section{Conflictos de interés}

Los autores declaran no tener conflictos de interés durante el planteamiento, ejecución de la investigación y la elaboración del artículo para su publicación.

Fecha de recepción: 12 de noviembre de 2015

Fecha de aceptación: 23 de noviembre de 2015 